\title{
Soft structures of $\gamma$-ray strength functions studied with the Oslo method
}

\author{
A. C. Larsen ${ }^{1, a}$, U. Agvaanluvsan ${ }^{2,3}$, L. A. Bernstein ${ }^{4}$, A. Bürger ${ }^{1}$, R. Chankova ${ }^{5}$, S. Goriely ${ }^{6}$, M. Guttormsen ${ }^{1}$, \\ T. Lönnroth ${ }^{7}$, G. E. Mitchell ${ }^{5}$, H. T. Nyhus ${ }^{1}$, A. Schiller ${ }^{8}$, S. Siem ${ }^{1}$, N. U. H. Syed ${ }^{1}$, H. K. Toft ${ }^{1}$, and A. Voinov ${ }^{8}$ \\ 1 Department of Physics, University of Oslo, N-0316 Oslo, Norway \\ 2 Stanford University, Palo Alto, California 94305 USA \\ 3 MonAme Scientific Research Center, Ulaanbaatar, Mongolia \\ 4 Lawrence Livermore National Laboratory, Livermore, California 94551, USA \\ 5 Department of Physics, North Carolina State University, Raleigh, North Carolina 27695, USA \\ 6 Institut d'Astronomie et d'Astrophysique, Université Libre de Bruxelles, CP 226, 1050 Brussels, Belgium \\ 7 Department of Physics, Åbo Akademi, FIN-20500 Åbo, Finland \\ 8 Department of Physics, Ohio University, Athens, OH 45701, USA
}

\begin{abstract}
We present experimental $\gamma$-ray strength functions up to $E_{\gamma} \sim S_{n}$ measured at the Oslo Cyclotron Laboratory for several Sc, V, Mo, and Sn isotopes. For the lighter nuclei, an unexpected enhancement of the strength function at low $\gamma$-ray energies has been revealed. This enhancement could potentially have an impact on neutron-capture cross sections for unstable, neutron-rich nuclei. For the Sn isotopes, we observe increased strength around the neutron separation energy $S_{n}$.
\end{abstract}

\section{Introduction}

The so-called $\gamma$-ray strength function characterizes average decay properties of excited nuclei. This quantity is very important for describing the $\gamma$-emission channel in nuclear reactions. It is also indispensable for calculating nuclear reaction cross sections and reaction rates relevant for, e.g., astrophysical applications.

The nuclear physics group at the Oslo Cyclotron Laboratory (OCL) has developed a unique method where both nuclear level density and gamma-ray strength function can be extracted simultaneously from primary $\gamma$-ray spectra (see, e.g., Ref. [1] and references therein).

In this work we present recent results on the $\gamma$-ray strength functions of the nuclei ${ }^{44,45} \mathrm{Sc}[1],{ }^{50,51} \mathrm{~V}$ [2], ${ }^{93-98} \mathrm{Mo}$ [3], and ${ }^{116-119} \mathrm{Sn}[4,5]$.

\section{Experiments}

The experiments were conducted at the OCL utilizing a beam of ${ }^{3} \mathrm{He}$ particles bombarding the self-supporting targets, with typical isotopic enrichment of $\geq 95 \%$. Particle- $\gamma$ coincidences were measured with eight $\Delta E-E$ Si particle telescopes and the $\gamma$-detector array CACTUS. The reactions selected by gating on the outgoing particle species, were the neutron pickup $\left({ }^{3} \mathrm{He}, \alpha \gamma\right)$ and the inelastic scattering $\left({ }^{3} \mathrm{He},{ }^{3} \mathrm{He}^{\prime} \gamma\right)$. From the reaction kinematics, the excitation energy $E_{x}$ of the product nucleus is given; thus, a unique $\gamma$-ray spectrum could be assigned for each excitation energy up to the neutron (proton) separation energy.

\footnotetext{
a e-mail: a.c.larsen@fys.uio.no
}

These $\gamma$-ray spectra, after correcting for the response function of CACTUS, were used for extracting the first $\gamma$-rays emitted in the decay cascades. The resulting primary $\gamma$-ray spectra for each $E_{x}$ give information on the nuclear level density (NLD) and the $\gamma$-ray strength function $(\gamma \mathrm{SF})$. The $E_{x}$ versus $E_{\gamma}$ matrix $P\left(E_{x}, E_{\gamma}\right)$ of primary $\gamma$-ray spectra depends on two functions: the NLD $\left(\rho\left(E_{x}\right)\right)$ and the $\gamma \mathrm{SF}$ $\left(f\left(E_{\gamma}\right)\right)$. The latter is a function of the $\gamma$-ray energy only according to the Brink hypothesis [6]:

$$
P\left(E_{x}, E_{\gamma}\right) \propto \rho\left(E_{x}-E_{\gamma}\right) f\left(E_{\gamma}\right) E_{\gamma}^{3} .
$$

Thus, the whole landscape $P\left(E_{x}, E_{\gamma}\right)$ is described by these two vectors. For further details on the simultaneous determination of NLD and $\gamma \mathrm{SF}$, see [7].

\section{Results for Sc, V, and Mo}

The measured $\gamma$-ray strength functions of ${ }^{44,45} \mathrm{Sc}$ and ${ }^{50,51} \mathrm{~V}$ are shown in Fig. 1, while the Mo $\gamma$ SFs are displayed in Fig. 2.

We see that all the $\gamma \mathrm{SF}$ increase with increasing $\gamma$-ray energy for $E_{\gamma}>4 \mathrm{MeV}$. This is expected as this region is dominated by the low-energy tail of the giant dipole resonance (GDR); also, at these energies the models describe the data well. However, for $E_{\gamma}<4 \mathrm{MeV}$, a puzzling feature is observed: the $\gamma \mathrm{SF}$ increase with decreasing $\gamma$-ray energy making a U-like shape of the $\gamma$ SFs.

As this "upbend" behavior is not predicted by any theory, the Hybrid model [8] and the Generalized Lorentzian 

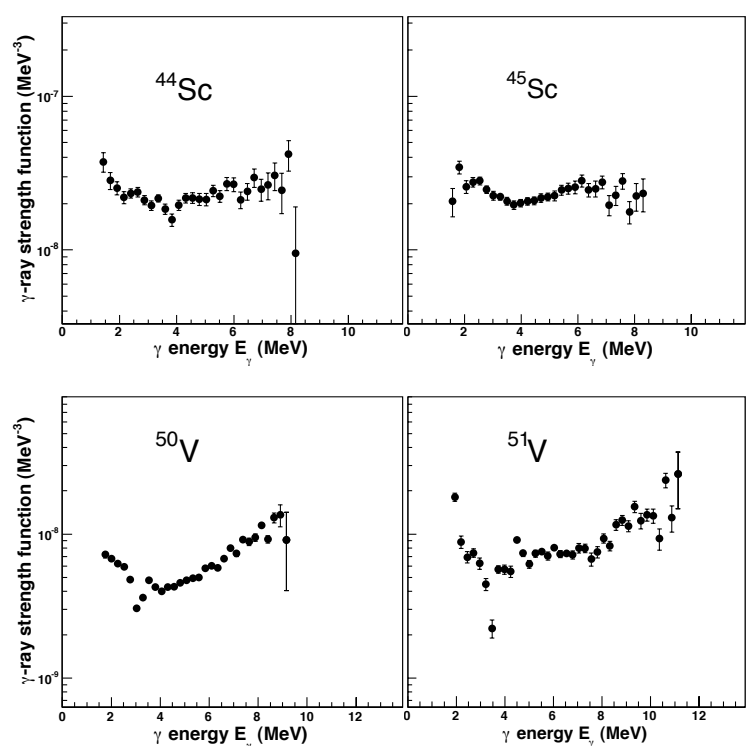

Fig. 1. Gamma-ray strength functions of ${ }^{44,45} \mathrm{Sc}[1]$ and ${ }^{50,51} \mathrm{~V}[2]$.

(GLO) model ([9] and references therein) have been modified in order to reproduce the Mo data reasonably well using a constant temperature of the final states $(T=$ const.) [12].

Using the models shown in Fig. 2 in addition to the same models using a variable temperature of the final states $\left(T \sim \sqrt{E_{f}}\right)$, the Maxwellian averaged neutron-capture reaction rates have been calculated for a temperature of $T=$ $10^{9} \mathrm{~K}$ typical of the r-process nucleosynthesis [10] with the code TALYS [11]. The ratios of the rates using the GLO (or Hybrid) model with and without the upbend for Fe, Mo, and $\mathrm{Cd}$ are displayed in Figs. 3 and 4 [12]. We observe that close to the stability line the upbend structure has a relatively small influence. However, for exotic neutron-rich nuclei the impact may become large, essentially due to the low neutron separation energies allowing only for $\gamma$-decays with energies lower than typically $2 \mathrm{MeV}$. In this case, the strength in the upbend structure dominates the decay. These calculations show that a proper understanding of the $E_{\gamma} \rightarrow 0$ limit of the RSF can be of crucial importance in the determination of radiative neutron capture cross section for exotic neutron-rich nuclei. This effect could have a non-negligible impact on the neutron captures that can potentially take place in astrophysical environments characterized by high neutron densities, in particular during the r-process nucleosynthesis. For more details, see [12].

\section{Results for the Sn isotopes}

The $\gamma \mathrm{SFs}$ of ${ }^{116-119} \mathrm{Sn}$ are displayed in Fig. 5. Here, there is no sign of a low-energy increase observed in the lighter nuclei. However, another feature strikes the eye: at about $E_{\gamma}=5 \mathrm{MeV}$, a change in slope takes place. This could indicate that some sort of resonance might be present. Indeed, for the neutron-rich isotopes ${ }^{129-133} \mathrm{Sn}$ and ${ }^{133,134} \mathrm{Sb}$,

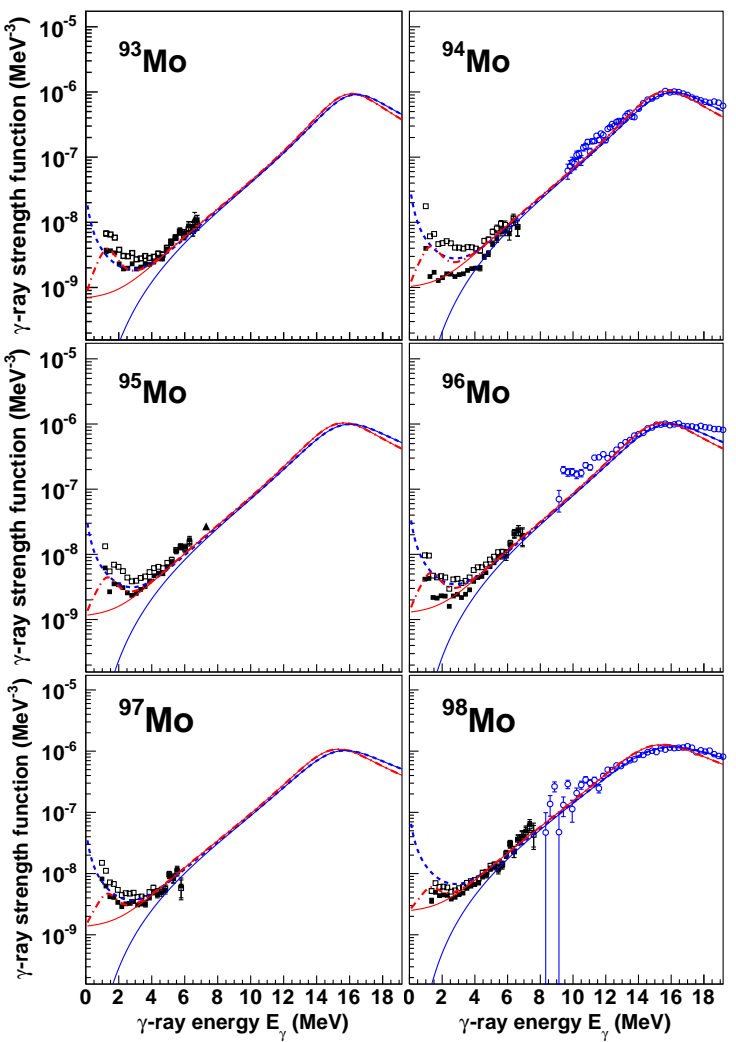

Fig. 2. Gamma-ray strength functions of ${ }^{93-98} \mathrm{Mo}[3,12]$. Gamma-ray strength functions for ${ }^{93-98}$ Mo. Experimental data points with the normalization of Ref. [3] are shown as open squares. The filled squares are obtained when normalizing the experimental NLDs to the calculations of [13]. Giant resonance photoabsorption data (blue open circles) for ${ }^{94,96,98} \mathrm{Mo}$ are taken from [14]. The black triangles represent measured $E 1$ strengths for ${ }^{93,95}$ Mo from [9]. The blue dashed line corresponds to the Hybrid model modified to reproduce the upbend $[8,12]$ and the blue solid line to the Hybrid model with zero temperature $(T=$ $0 \mathrm{MeV})$. The red solid line shows the Generalized Lorentzian (GLO) model (see [9] and references therein) with $T=0.3 \mathrm{MeV}$, while the red, dashed-dotted line is the GLO with a Lorentzian resonance centered at $E_{\gamma}=1.5 \mathrm{MeV}$.

an $E 1$-type pygmy resonance has been observed at $\gamma$-ray energies around $8-10 \mathrm{MeV}[15,16]$. This has been interpreted as excess neutrons oscillating against the core nucleons. Also from nuclear resonance fluorescence (NRF) experiments, there are strong indications of an increased $E 1$ strength for $E_{\gamma}=6-8 \mathrm{MeV}$ for stable even-even tin isotopes $[17,18]$. However, since the present experimental technique does not distinguish between electric and magnetic transitions, the enhanced strength seen in the Oslo data could in principle be due to both E1- and M1-type radiation.

For ${ }^{117} \mathrm{Sn}$, we have compared our results with $(\gamma, n)$ cross-section data, in addition to the GLO model and QRPA calculations, see Ref. [19] for details. This is shown in Fig. 6. It is clear from our analysis that extra strength is present below and above the neutron threshold in ${ }^{117} \mathrm{Sn}$. It 


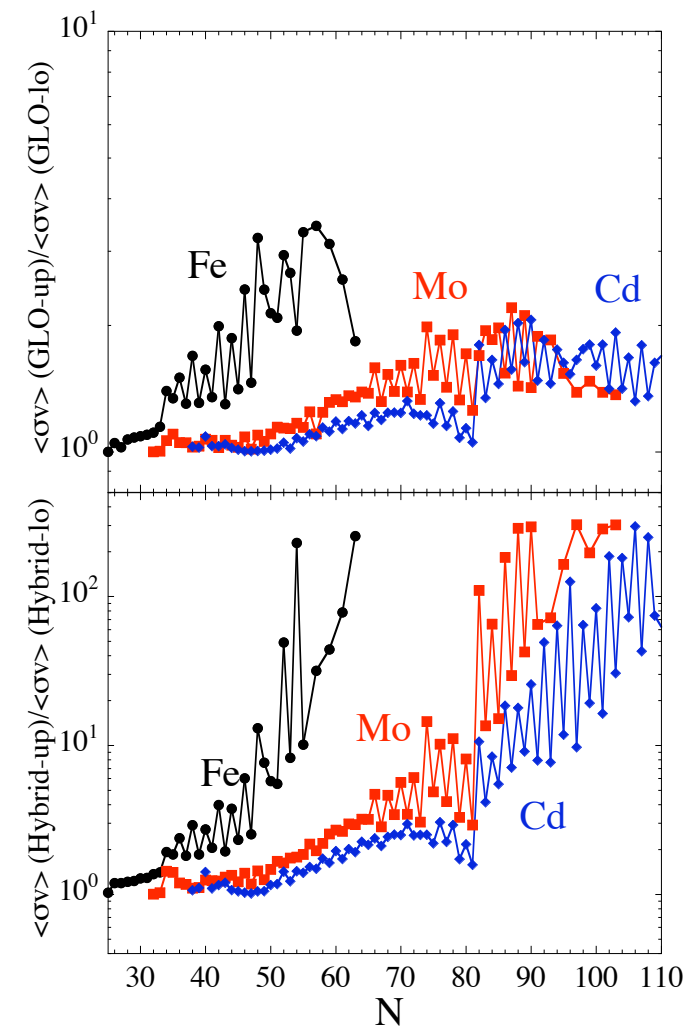

Fig. 3. Ratios of Maxwellian-averaged $(n, \gamma)$ reaction rates at $T=$ $10^{9} \mathrm{~K}$ with and without the inclusion of the upbend structure for the $\mathrm{Fe}$, Mo and $\mathrm{Cd}$ isotopic chains up to the neutron drip line. The upper panel compares the GLO models and the lower panel the Hybrid models. A constant temperature of final states is applied for all models.

is also seen that the standard Lorentzian (SLO) model fits the $(\gamma, x)$ data very well; however, it is not able to describe neither the shape nor the magnitude of our data below the neutron threshold.

\section{Conclusion}

The nuclear physics group at the OCL has developed the so-called Oslo method in order to measure level density and $\gamma$-ray strength up to the nucleon separation energy. The $\gamma \mathrm{SF}$ of several Sc, V, Mo, and $\mathrm{Sn}$ isotopes have been presented. For the lighter nuclei, the still unexplained lowenergy enhancement is observed. This feature could potentially have a significant influence on the Maxwellianaveraged reaction rates.

For the heavier Sn isotopes, there is no upbend but some additional strength seems to be present for $E_{\gamma}>5$ $\mathrm{MeV}$, that could possibly be an $E 1$ pygmy resonance due to neutron-skin oscillations. Further investigations are needed both on the upbend phenomenon and the resonance-like structure of the $\mathrm{Sn}$ isotopes.

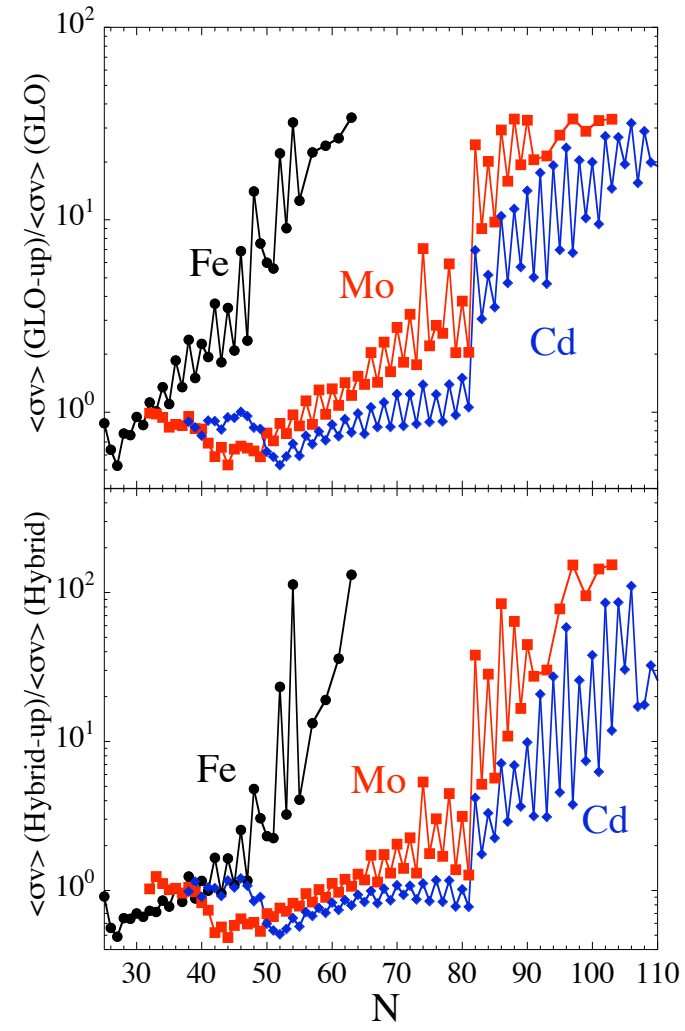

Fig. 4. Same as Fig. 3 for the comparison between the GLO models (upper panel) and the Hybrid models (lower panel) using a variable temperature of final states.

\section{Acknowledgments}

The authors wish to thank E. A. Olsen and J. Wikne for excellent experimental conditions. Financial support from the Norwegian Research Council and the Department of Physics, University of Oslo, is gratefully acknowledged.

\section{References}

1. A. C. Larsen, M. Guttormsen, R. Chankova, F. Ingebretsen, T. Lönnroth, S. Messelt, J. Rekstad, A. Schiller, S. Siem, N. U. H. Syed, and A. Voinov, Phys. Rev. C 76, 044303 (2007).

2. A. C. Larsen, R. Chankova, M. Guttormsen, F. Ingebretsen, T. Lönnroth, S. Messelt, J. Rekstad, A. Schiller, S. Siem, N. U. H. Syed, A. Voinov, and S. W. Ødegård, Phys. Rev. C 73, 064301 (2006).

3. M. Guttormsen, R. Chankova, U. Agvaanluvsan, E. Algin, L.A. Bernstein, F. Ingebretsen, T. Lönnroth, S. Messelt, G.E. Mitchell, J. Rekstad, A. Schiller, S. Siem, A.C. Sunde, A. Voinov and S. Ødegård, Phys. Rev. C 71, 044307 (2005). 


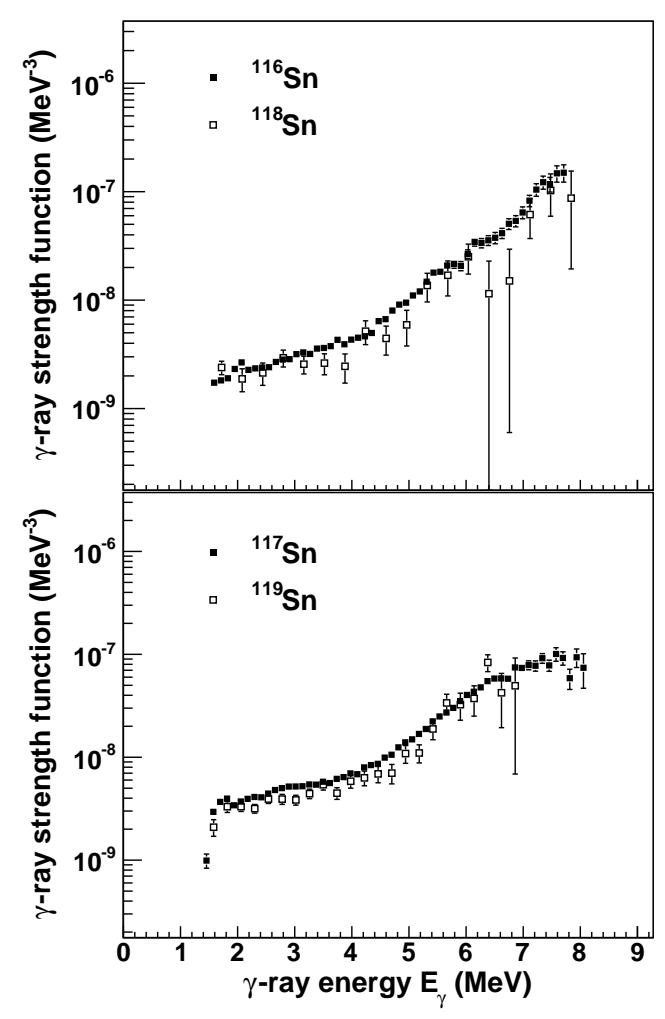

Fig. 5. Gamma-ray strength functions of ${ }^{116,118} \mathrm{Sn}$ (upper panel) and ${ }^{117,119} \mathrm{Sn}$ (lower panel). The strength functions of ${ }^{116,118,119} \mathrm{Sn}$ are preliminary [5].

4. U. Agvaanluvsan, A. C. Larsen, R. Chankova, M. Guttormsen, G. E. Mitchell, A. Schiller, S. Siem, and A. Voinov, Phys. Rev. Lett. 102,162504 (2009).

5. H. K. Toft, Ph.D thesis (work in progress).

6. D.M. Brink, PhD thesis, Oxford University (1955).

7. A. Schiller, L. Bergholt, M. Guttormsen, E. Melby, J. Rekstad, S. Siem, Instrum. Methods Phys. Res. A 447 494 (2000).

8. S. Goriely, Phys. Lett. B436, 10 (1998).

9. T. Belgya, O. Bersillon, R. Capote, T. Fukahori, G. Zhigang, S. Goriely, M. Herman, A. V. Ignatyuk, S. Kailas, A. Koning, P. Oblozinsky, V. Plujko and P. Young, Handbook for calculations of nuclear reaction data, RIPL2. IAEA-TECDOC-1506 (IAEA, Vienna, 2006); also available online at http://www-nds.iaea.org/RIPL-2/

10. M. Arnould, S. Goriely, and K. Takahashi, Phys. Rep. 450, 97 (2007).

11. A. J. Koning, S. Hilaire, and M. C. Duijvestijn, "TALYS-1.0", in Proceedings of the International Conference on Nuclear Data for Science and Technology, April 22-27, 2007, Nice, France. Editors: O. Bersillon, F. Gunsing, E. Bauge, R. Jacqmin, and S. Leray, EDP Sciences, 211 (2008).

12. A. C. Larsen and S. Goriely, submitted to Phys. Rev. C.

13. S. Goriely, S. Hilaire, and A.J. Koning, Phys. Rev. C 78, 064307 (2008).

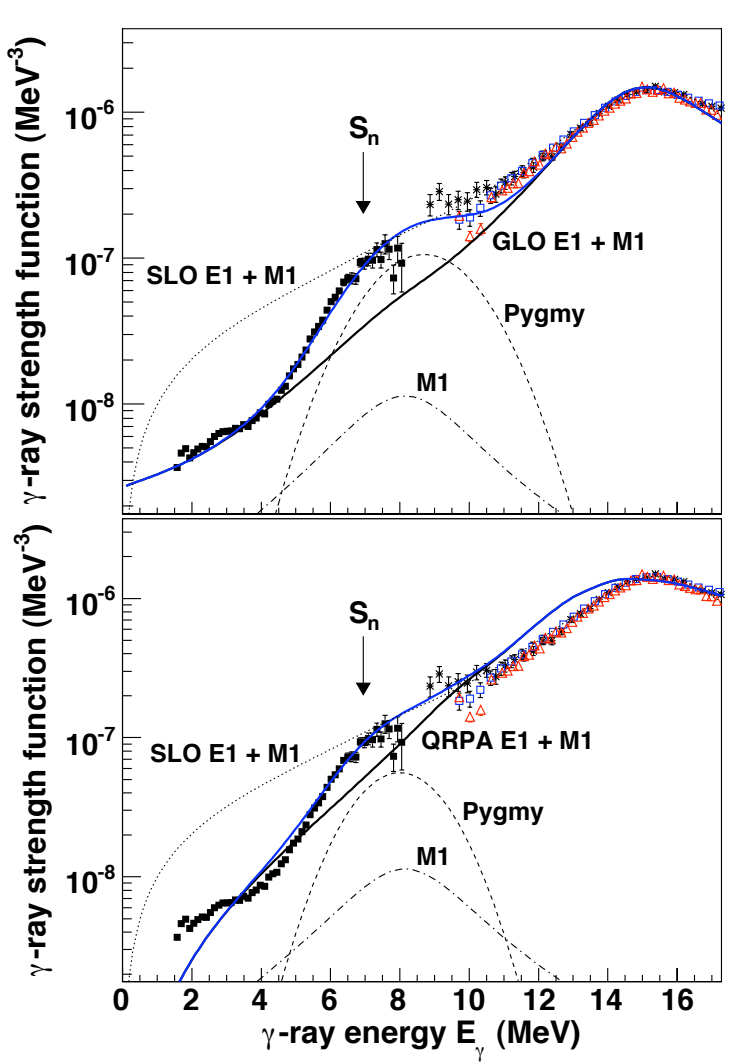

Fig. 6. Upper panel: Gamma-ray strength function of ${ }^{117} \mathrm{Sn}$ (black squares) [19], and from $(\gamma, x)$ photonuclear reactions (red open triangles [20], stars [21], and blue open squares [22]). The sum of the GLO $E 1$ strength and the $M 1$ spin-flip resonance is shown as a solid line. The GLO E1 strength, the $M 1$ spin-flip resonance (dashed-dotted line), and a Gaussian parameterization of the pygmy resonance (dashed line) are added to get a best fit (thick, blue solid line) to the data. The sum of the SLO E1 strength and the $M 1$ strength is shown for comparison (dotted line). Lower panel: same as in the upper panel except that a QRPA $E 1$ strength is used [23].

14. H. Beil, R. Bergère, P. Carlos, A. Leprêtre, A. De Miniac, A. Veyssière, Nucl. Phys. A227, 427 (1974).

15. P. Adrich et al., Phys. Rev. Lett. 95, 132501 (2005).

16. A. Klimkiewicz et al., Phys. Rev. C. 76, 051603(R) (2007).

17. K. Govaert et al., Phys. Rev.C 57, 2229 (1998).

18. A. Tonchev, private communication.

19. U. Agvaanluvsan, A. C. Larsen, R. Chankova, M. Guttormsen, G. E. Mitchell, A. Schiller, S. Siem, and A. Voinov, Phys. Rev. Lett. 102, 162504 (2009).

20. S. C. Fultz et al., Phys. Rev. 186, 1255 (1969).

21. A. Leprêtre et al., Nucl. Phys. A219, 39 (1974).

22. V. V. Varlamov et al., Preprint No 2003-2/715, MSU SINP (Moscow, 2003); B. S. Ishkhanov and V. V. Varlamov, Phys. At. Nucl. 67, 1664 (2004).

23. S. Goriely and E. Khan, Nucl. Phys. A706, 217 (2002). 